Groups Geom. Dyn. 4 (2010), 813-833

DOI $10.4171 / \mathrm{GGD} / 108$
Groups, Geometry, and Dynamics

(C) European Mathematical Society

\title{
The conjugacy problem in the Grigorchuk group is polynomial time decidable
}

\author{
Igor Lysenok, Alexei Myasnikov and Alexander Ushakov*
}

\begin{abstract}
In this paper we prove that the conjugacy problem in the Grigorchuk group $\Gamma$ has polynomial time complexity. This solves a problem posed by Grigorchuk rather unexpectedly.
\end{abstract}

Mathematics Subject Classification (2010). 20F10, 20 E08.

Keywords. Conjugacy problem, Grigorchuk group.

\section{Introduction}

In this paper we discuss algorithmic complexity of the conjugacy problem in the original Grigorchuk group $\Gamma$. The group $\Gamma$ first appeared in [5] almost thirty years ago, now it plays an important part in several areas of modern group theory: growth in groups [6], Burnside problems [5], amenability [7], just infinite groups [8]. Recently the group $\Gamma$ was proposed as a possible platform for cryptographic schemes (see [4], [15], [14]), where the algorithmic security of the schemes is based on the computational hardness of certain variations of the word and conjugacy problems in $\Gamma$. The bibliography on $\Gamma$ is quite extensive; here we refer to [10] and [9], which provide a comprehensive and accessible survey on $\Gamma$.

Our interest in $\Gamma$ comes from a rather different direction: it is concerned with the foundations of algorithmic group theory. Recall that the classical approach to algorithmic problems in groups deals mostly with finitely presented groups - an old tradition, coming from topology. Another way to study algorithmic problems in groups stems from constructive mathematics, where the elements of a group must be given as finitary objects (matrices over number fields, automorphisms of graphs, complexes, or other constructible objects) and the group multiplication has to be effectively described or is computable. Rabin's recursive groups [16] or Malcev's constructible groups [13] provide typical examples of this type. A more general approach to algorithmic group theory is concerned with groups given by arbitrary recursive presentations. There are some known general results in this direction,

\footnotetext{
${ }^{*}$ The work of the second and the third author was partially supported by the NSF grant DMS-0914773.
} 
including the spectacular Higman embedding theorem [11], but a cohesive theory is lacking (perhaps due to the huge variety of groups in this class). The Grigorchuk group $\Gamma$ may serve as a case study here. Indeed, $\Gamma$ can be easily described as generated by four particular automorphisms of the infinite rooted binary tree, but it is not finitely presented, though it has a nice infinite recursive "self-similar" presentation. Studying algorithmic problems in $\Gamma$ may provide some interesting insight on how to deal with recursively presented groups whose presentations are infinite but can be described by repeating some typical finite pattern or obvious self-similarity.

The word, conjugacy, and isomorphism problems are the three famous algorithmic problems in group theory raised by Max Dehn in 1911. The word problem (WP) in $\Gamma$ is decidable and its time complexity is $O(n \log n)$ (see, for example, [10], [9]). It has been shown in [12], [17] that the conjugacy problem (CP) is decidable in $\Gamma$. In fact, $\Gamma$ is conjugacy separable [18]. Moreover, [12] gives a complete characterization of the Grigorchuk groups $G_{\omega}$ with decidable CP: these are precisely those where the sequence $\omega$ is recursive. Another decision algorithm for $\mathrm{CP}$ in $\Gamma$ is described in [2] and [9]. This is a branching algorithm, it is based on a branching rewriting process, similar to the original decision algorithm for the word problem in $\Gamma$ [5]. The time upper bound for this algorithm given in [9] is double exponential. This raises a natural question (see Problem 5.1 in [9]): what is the time complexity of $\mathrm{CP}$ in $\Gamma$ ?

We show below that $\mathrm{CP}$ in $\Gamma$ can be solved in polynomial time. To prove this we modify the decision algorithm from [9]: given two elements $u, v \in \Gamma$ we first construct a unique conjugacy tree $T_{u, v}$ (there are exponentially many trees in [9]), then we provide a routine, similar to the one in [9], which given a conjugacy tree $T_{u, v}$ checks whether or not $u$ and $v$ are conjugate in $G$. This routine requires polynomial time in the size of $T_{u, v}$. Finally, we show, and this the main technical result of the paper, that the size of $T_{u, v}$ is polynomial in the total length $|u|+|v|$, so the decision algorithm is polynomial in time. This part is tricky, to prove it we replace, following [1], the standard length on $\Gamma$ by a new "weighted" length, called the norm, and show that the standard splitting $w \rightarrow\left(w_{0}, w_{1}\right)$ of elements from $\operatorname{St}_{\Gamma}(1)$ has very nice metric properties relative to the norm. These metric properties allow one to prove that the length of the elements that appear in the construction of $T_{u, v}$ drops exponentially, so the height of the tree $T_{u, v}$ is about $\log (|u|+|v|)$, hence the size of $T_{u, v}$ is polynomial in $|u|+|v|$. The degree $d$ of the polynomial depends on the metric properties of the splitting, currently $d=7$. The resulting decision algorithm for $\mathrm{CP}$ in $\Gamma$ has the upper time bound $O\left(n^{8}\right)$. We would like to point out that it is not clear whether this upper bound is tight or not. In fact, all our computer experiments indicate that the algorithm is quite practical, it behaves like an algorithm with a quadratic time upper bound. The algorithm itself is available online [3]. Finally, we want to mention that it seems plausible that a similar method could give a polynomial time decision algorithm for $\mathrm{CP}$ in some other self-similar contracting groups. 


\section{Preliminaries on the Grigorchuk group}

In this section, following [10] and [9], we define the Grigorchuk group $\Gamma$ and recall some of its properties. Notation and the techniques introduced here are heavily used throughout the paper.

2.1. The Grigorchuk group $\Gamma$. For a set $X$ we denote by $X^{*}$ the set of all finite words (sequences) in $X$. If $u \in X^{*}$ and $x \in X$ then $|u|_{x}$ is the number of occurrences of $x$ in $u$ and $|u|$ is the length of $u$.

Let $\mathcal{T}$ be an infinite rooted regular binary tree. Recall that the vertex set of $\mathcal{T}$ is precisely the set $\{0,1\}^{*}$ of all finite binary words (the empty word $\varepsilon$ at the root) and two words $u$ and $v$ are connected by an edge in $\mathcal{T}$ if and only if one of them, say $v$, is obtained from the other by adding one bit $b \in\{0,1\}$ at the end, so $v=u b$. The tree $\mathcal{T}$ is shown in Figure 1.

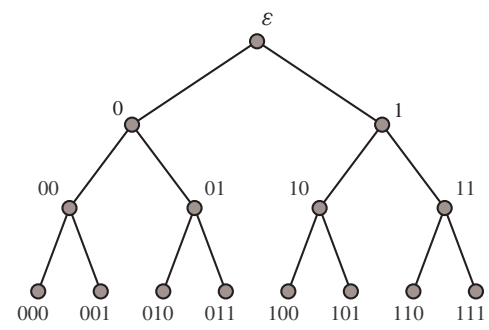

Figure 1. Binary tree with labeled vertices.

Let $\operatorname{Aut}(\mathcal{T})$ be the group of automorphisms of $\mathcal{T}$ as a rooted tree. Note that any automorphism of $\mathcal{T}$ fixes the root $\varepsilon$. Clearly, every $\phi \in \operatorname{Aut}(\mathcal{T})$ either fixes the vertices 0,1 or permutes them. The ones that fix 0 and 1 form a normal subgroup $\operatorname{St}(1)$ of $\operatorname{Aut}(\mathcal{T})$ of index 2. Let $T_{0}$ be the "left" subtree of $\mathcal{T}$, i.e., the subgraph induced by all vertices that start with 0 , and $T_{1}$ the "right" subtree of $\mathcal{T}$ induced by all vertices starting with 1 . The automorphism $a \in \operatorname{Aut}(\mathcal{T})$, defined on vertices of $\mathcal{T}$ by

$$
a\left(b_{1}, b_{2}, \ldots, b_{n}\right)=\left(1-b_{1}, b_{2}, \ldots, b_{n}\right),
$$

swaps the subtrees $T_{0}$ and $T_{1}$, hence $a \notin \operatorname{St}(1)$ and $\operatorname{Aut}(\mathcal{T})=\operatorname{St}(1) \sqcup \operatorname{St}(1) a$.

The Grigorchuk group $\Gamma$ is the subgroup of $\operatorname{Aut}(\mathcal{T})$ generated by four automorphisms $a, b, c, d$, where $b, c, d \in \operatorname{St}(1)$ are defined recursively as follows:

$$
b\left(b_{1}\right)=b_{1}, \quad c\left(b_{1}\right)=b_{1}, \quad d\left(b_{1}\right)=b_{1}
$$


and

$$
\begin{aligned}
& b\left(b_{1}, b_{2}, \ldots, b_{n}\right)= \begin{cases}\left(b_{1}, 1-b_{2}, b_{3}, \ldots, b_{n}\right) & \text { if } b_{1}=0, \\
\left(b_{1}, c\left(b_{2}, \ldots, b_{n}\right)\right) & \text { if } b_{1}=1 ;\end{cases} \\
& c\left(b_{1}, b_{2}, \ldots, b_{n}\right)= \begin{cases}\left(b_{1}, 1-b_{2}, b_{3}, \ldots, b_{n}\right) & \text { if } b_{1}=0, \\
\left(b_{1}, d\left(b_{2}, \ldots, b_{n}\right)\right) & \text { if } b_{1}=1 ;\end{cases} \\
& d\left(b_{1}, b_{2}, \ldots, b_{n}\right)= \begin{cases}\left(b_{1}, b_{2}, \ldots, b_{n}\right) & \text { if } b_{1}=0, \\
\left(b_{1}, b\left(b_{2}, \ldots, b_{n}\right)\right) & \text { if } b_{1}=1 .\end{cases}
\end{aligned}
$$

It is easy to see that the automorphisms $a, b, c, d$ satisfy the relations

$$
a^{2}=b^{2}=c^{2}=d^{2}=1, \quad b c=c b=d .
$$

In particular,

$$
\langle a\rangle \simeq \mathbb{Z}_{2} \text { and }\langle b, c, d\rangle \simeq \mathbb{Z}_{2} \times \mathbb{Z}_{2}
$$

Consider the group

$$
\Gamma_{0}=\left\langle a, b, c, d \mid a^{2}=b^{2}=c^{2}=d^{2}=1, b c=c b=d\right\rangle .
$$

The group $\Gamma_{0}$ is the free product of the subgroups $\langle a\rangle$ and $\langle b, c, d\rangle$. Let $X=$ $\{a, b, c, d\}$. It follows that any word $w \in\left(X \cup X^{-1}\right)^{*}$ is equal in $\Gamma_{0}$ to a unique reduced word

$$
\operatorname{red}(w)=u_{0} a u_{1} \ldots u_{n} a u_{n+1},
$$

where $u_{1}, \ldots, u_{n} \in\{b, c, d\}, u_{0}, u_{n+1} \in\{\varepsilon, b, c, d\}$. In particular, $u_{0}$ and $u_{n+1}$ could be empty. The following rewriting system, $\mathcal{W}$,

$$
\begin{gathered}
x^{2} \rightarrow \varepsilon, \quad x^{-1} \rightarrow x \quad(x \in X), \\
r s \rightarrow t \quad(r, s, t \in\{b, c, d\}, r \neq s \neq t)
\end{gathered}
$$

is terminating and confluent, and $\operatorname{red}(w)$ is precisely the reduced form of $w$ relative to $\mathcal{W}$. Clearly, $|\operatorname{red}(w)| \leq|w|$. Furthermore, given a word $w \in\left(X \cup X^{-1}\right)^{*}$ one can compute red $(w)$ in time $O(|w|)$.

From now on we only consider words over $X$. Denote by $\mathcal{R}$ the set of all reduced words in $X^{*}$ and by $\mathcal{R}^{e}$ the set of all reduced words $w$ in $X^{*}$ such that $|w|_{a}$ is even.

Let $\operatorname{St}_{\Gamma}(1)=\operatorname{St}(1) \cap \Gamma$ be the set of automorphisms in $\Gamma$ stabilizing the first level of $\mathcal{T}$, i.e., stabilizing the vertices $\{0,1\}$.

Lemma 2.1. The following holds:

1) A word $w \in X^{*}$ represents an element of $\mathrm{St}_{\Gamma}(1)$ if and only if $|w|_{a}$ is even.

2) $\Gamma=\operatorname{St}_{\Gamma}(1) \sqcup a \operatorname{St}_{\Gamma}(1)$.

3) $\operatorname{St}_{\Gamma}(1)=\langle b, c, d, a b a, a c a, a d a\rangle$. 
Every automorphism $g \in \operatorname{St}(1)$ fixes the first level of $\mathcal{T}$ and hence induces automorphisms $g_{0}$ and $g_{1}$ on the subtrees $T_{0}$ and $T_{1}$ of $\mathcal{T}$. Since the subtrees $T_{0}$ and $T_{1}$ are naturally isomorphic to $\mathcal{T}$, the mapping $g \mapsto\left(g_{0}, g_{1}\right)$ gives a group isomorphism

$$
\psi: \operatorname{St}(1) \rightarrow \operatorname{Aut}(\mathcal{T}) \times \operatorname{Aut}(\mathcal{T})
$$

and, hence, in the event $g \stackrel{\psi}{\mapsto}\left(g_{0}, g_{1}\right)$ we can write $g=\left(g_{0}, g_{1}\right)$.

If $g, h \in \mathrm{St}(1)$ and $g=\left(g_{0}, g_{1}\right)$ and $h=\left(h_{0}, h_{1}\right)$ then (since $\psi$ is an isomorphism)

$$
g h=\left(g_{0} h_{0}, g_{1} h_{1}\right)
$$

and (by an easy computation)

$$
a^{-1} g a=\left(g_{1}, g_{0}\right)
$$

We use these formulas frequently and without references. Also, it is easy to check that for generators $b, c, d$ the following equalities hold:

$$
\begin{aligned}
b & =(a, c), & a b a & =(c, a), \\
c & =(a, d), & a c a & =(d, a), \\
d & =(1, b), & a d a & =(b, 1) .
\end{aligned}
$$

Therefore, the restriction of $\psi$ to $\mathrm{St}_{\Gamma}(1)$ gives a monomorphism

$$
\psi: \operatorname{St}_{\Gamma}(1) \rightarrow \Gamma \times \Gamma,
$$

which is not onto (see [10] or [9]). If $g \in \mathrm{St}_{\Gamma}$ (1) is represented by a reduced word $w \in X^{*}$ then one can easily find the reduced forms of the automorphisms $g_{0}$ and $g_{1}$. Indeed, in this case one can assume that $w \in \mathcal{R}^{e}$ and is represented as a product

$$
w=u_{0} \cdot\left(a u_{1} a\right) \cdot u_{2} \cdot\left(a u_{3} a\right) \cdot u_{4} \ldots u_{k-2} \cdot\left(a u_{k-1} a\right) \cdot u_{k},
$$

where $u_{0}, \ldots, u_{k} \in\{b, c, d\}$ and $u_{0}, u_{k}$ are, possibly, trivial. We refer to these $u_{i}$ and $\left(a u_{j} a\right)$ as to the factors of $w$. Now define two mappings $\phi_{i}: \mathcal{R}^{e} \rightarrow \mathcal{R}, i=1,2$, inductively on the number of factors. First, define $\phi_{i}$ on the factors according to the formulas in (5):

$$
\begin{aligned}
\phi_{0}(b) & =a, & \phi_{0}(a b a) & =c, \\
\phi_{0}(c) & =a, & \phi_{0}(a c a) & =d, \\
\phi_{0}(d) & =\varepsilon, & \phi_{0}(a d a) & =b ; \\
\phi_{1}(b) & =c, & \phi_{1}(a b a) & =a, \\
\phi_{1}(c) & =d, & & \phi_{1}(a c a)=a, \\
\phi_{1}(d) & =b, & & \phi_{1}(a d a)=1 .
\end{aligned}
$$


Then define by induction

$$
w_{i}=\phi_{i}(w)=\operatorname{red}\left(\phi_{i}\left(v_{0}\right) \phi_{i}\left(v_{1} \ldots v_{k}\right)\right), \quad i=0,1,
$$

where $w=v_{0} \ldots v_{k}$ is the factor decomposition (6) of $w$. It follows immediately from the construction that

$$
w \stackrel{\psi}{\longmapsto}\left(w_{0}, w_{1}\right) .
$$

for any $w \in \mathcal{R}^{e}$. Notice that it takes time $O(|w|)$ to compute the pair $\left(w_{0}, w_{1}\right)$.

Lemma 2.2. Let $w \in \mathcal{R}^{e}$ and $w=\left(w_{0}, w_{1}\right)$. Then the following holds.

1) $\left|w_{0}\right|,\left|w_{1}\right| \leq \frac{|w|+1}{2}$.

2) Moreover, if $w$ starts with a then $\left|w_{0}\right|,\left|w_{1}\right| \leq \frac{|w|}{2}$.

Remark 2.3. Let $w \in \mathcal{R}^{e}$. Then conjugating, if necessary, $w$ by some of its initial segments and reducing the result, one either obtains $b, c, d$, or a word $w^{\prime} \in \mathcal{R}^{e}$ that begins with $a$ and does not end on $a$.

2.2. The word problem in $\boldsymbol{\Gamma}$. Following [10], [9], in this section we discuss an algorithm for the word problem in $\Gamma$. The algorithm is based on three observations:

- If $|w|_{a}$ is odd then $w \notin \operatorname{St}_{\Gamma}(1)$, hence $w \neq 1$ in $\Gamma$.

- If $|w|_{a}$ is even then $w \in \mathcal{R}^{e}$ and $w=\left(\phi_{0}(w), \phi_{1}(w)\right)$. Moreover, since $\psi$ is a monomorphism, we then have

$$
w={ }_{\Gamma} 1 \Longleftrightarrow \phi_{0}(w)=_{\Gamma} 1 \text { and } \phi_{1}(w)={ }_{\Gamma} 1 .
$$

(Here and below $w={ }_{\Gamma} 1$ means that $w=1$ in $\Gamma$.) Therefore, the word problem for $w$ reduces to the word problem for $\phi_{0}(w)$ and $\phi_{1}(w)$, i.e., the process splits or branches.

- If $w=\left(w_{0}, w_{1}\right)$ and $|w|>1$ then $\left|w_{0}\right|,\left|w_{1}\right|<|w|$. Thus, the process stops after finitely many steps.

It is convenient to visualize the corresponding algorithm as an algorithm that on an input $w \in X^{*}$ constructs a finite, labeled, rooted binary tree $T_{w}$.

Algorithm 2.4 (Constructing the decision tree $T_{w}$ ).

InPUT. $w \in X^{*}$.

Output. A finite labeled rooted binary tree $T_{w}$.

Computations.

A. (Initialization) Let $T_{0}$ be a rooted binary tree with a single vertex (the root) $w$.

B. (Verification) Let $T$ be a current rooted binary tree whose vertices are words in $X^{*}$ and some of them are marked by "yes" or "no". Let $u$ be an unmarked leaf in $T$. Then: 
Compute $|u|_{a}$. If $|u|_{a}$ is odd, then label $u$ by "no" and output the resulting tree as $T_{w}$.

Otherwise, compute red $(u)$ and take its conjugate red $(u)^{\prime}$ from Remark 2.3. If $\operatorname{red}(u)$ is empty then label $u$ by "yes" and go to step B. If $\left|\operatorname{red}(u)^{\prime}\right|=1$ then label $u$ with "no". Otherwise, go to C.

If there are no unmarked leaves in $T$ output $T$ as $T_{w}$.

C. (Splitting) Compute $\phi_{0}(u)$ and $\phi_{1}(u)$ and add them as the "left" and the "right" children of $u$. Go to B.

Proposition 2.5. For a given word $w \in X^{*}$ the height of the tree $T_{w}$ is at most $\log _{2}|w|+1$.

Proof. By Lemma 2.2 we have $\left|w_{i}\right| \leq|w| / 2, i=0,1$, for $w \in \mathcal{R}^{e}$ provided that it begins with $a$ but does not end on $a$. Hence, starting with $w$, the Algorithm 2.4 can make at most $\log _{2}|w|$ splittings since it does not split words of length at most 1 . The verification step does not increase the height.

The following result is known (see, for example, [10], [9]), but we need the proof for the reference sake.

Theorem 2.6 (Word problem). The computational complexity of the word problem for the group $\Gamma$ is bounded by $\mathrm{O}\left(n \log _{2} n\right)$.

Proof. The algorithm for WP in $\Gamma$ works as follows. Given $w \in X^{*}$ it computes first the decision tree $T_{w}$. If $T_{w}$ has a vertex marked by "no" then $w \neq 1$ in $\Gamma$, otherwise $w=1$ in $\Gamma$. By Proposition 2.5 the decision tree $T_{w}$ has at most $\log _{2}|w|$ levels. Hence, to estimate the time required for the algorithm to construct $T_{w}$ one needs only to bound the time required to construct an arbitrary level in $T_{w}$. The verification step, as well as the splitting step, at a leaf $u$ requires only linear time in $|u|$. The total length of the vertices at a given level in $T_{w}$ is at most $|w|$. Hence the upper time bound for the complexity is $O\left(|w| \log _{2}|w|\right)$, as claimed.

2.3. The subgroup $K$. As we mentioned above, the monomorphism $\psi: \operatorname{St}_{\Gamma}(1) \rightarrow$ $\Gamma \times \Gamma$ is not onto. In this section we describe a method how one can effectively check if a given pair $\left(w_{0}, w_{1}\right) \in \Gamma \times \Gamma$ has a pre-image under $\psi$, and, if so, how to compute it. We refer to [10], Sections VIII.30 and VIII.25, for details.

Let

$$
K=\langle a b a b\rangle^{\Gamma}
$$

be the normal subgroup of $\Gamma$ generated by the element $a b a b$. It turns out that $K$ has index 16 in $\Gamma$ and

$$
K=\langle a b a b, \text { badabada, abadabad }\rangle .
$$


The Schreier coset graph of $K$ is shown in Figure 2. We denote the coset representatives of $K$ in $\Gamma$ by $1=g_{0}, g_{1}, \ldots, g_{15}$ according to the numbers in Figure 2 . Observe that $K$ is a subgroup of $\mathrm{St}_{\Gamma}(1)$ of index 8 with coset representatives $g_{0}, g_{1}$, $g_{4}, g_{5}, g_{8}, g_{9}, g_{12}, g_{13}$.

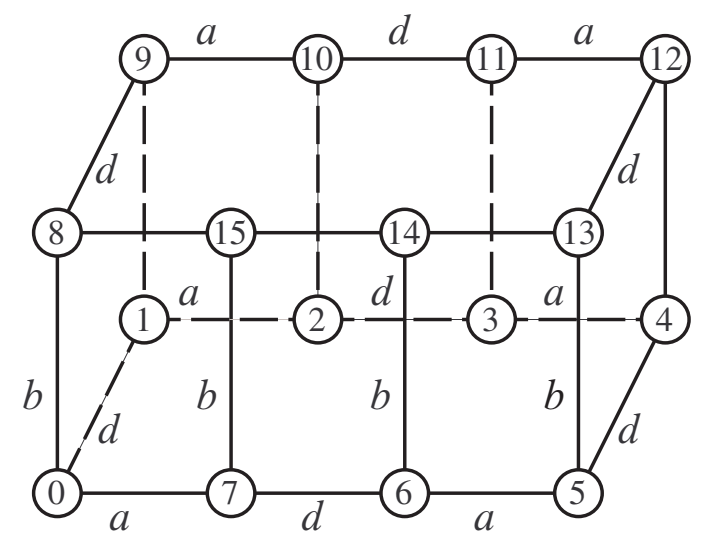

Figure 2. Schreier graph of $K \leq \Gamma$ relative to generators $\{a, b, d\}$.

Lemma 2.7. For any $k \in K$ there exist elements $u, v \in K$ such that $u=(k, 1)$ and $v=(1, k)$. In particular, $\psi(K) \geq K \times K$.

Proof. It is sufficient to prove the statement for the generators abab, badabada, abadabad of the subgroup $K$. A straightforward verification shows that

$$
\begin{aligned}
b \cdot a d a \cdot b \cdot a d a & =(\text { abab }, 1), \\
b a d a b \cdot a c a \cdot b a d a b \cdot a c a & =(\text { abadabad }, 1), \\
c \cdot b a d a b \cdot a c a \cdot b a d a b \cdot a c a \cdot c & =(\text { badabada }, 1) .
\end{aligned}
$$

Note that the words $w$ on the left-hand side of these equalities represent elements from $K$. Indeed, starting at the vertex 0 and reading such a word $w$ in the Schreier graph above (beforehand replacing $c$ with $b d$ ) one ends up again at 0 , thus proving the claim.

Lemma 2.8. Let $D=\langle(1, d),(1, a)\rangle \leq \Gamma \times \Gamma$. Then

1) $D$ is isomorphic to the dihedral group of order 8 ,

2) $\Gamma \times \Gamma=\psi\left(\operatorname{St}_{\Gamma}(1)\right) \rtimes D$.

Proof. See [10], p. 229.

The Schreier coset graph of $\psi\left(\operatorname{St}_{\Gamma}(1)\right) \leq \Gamma \times \Gamma$ is shown in Figure 3 . 


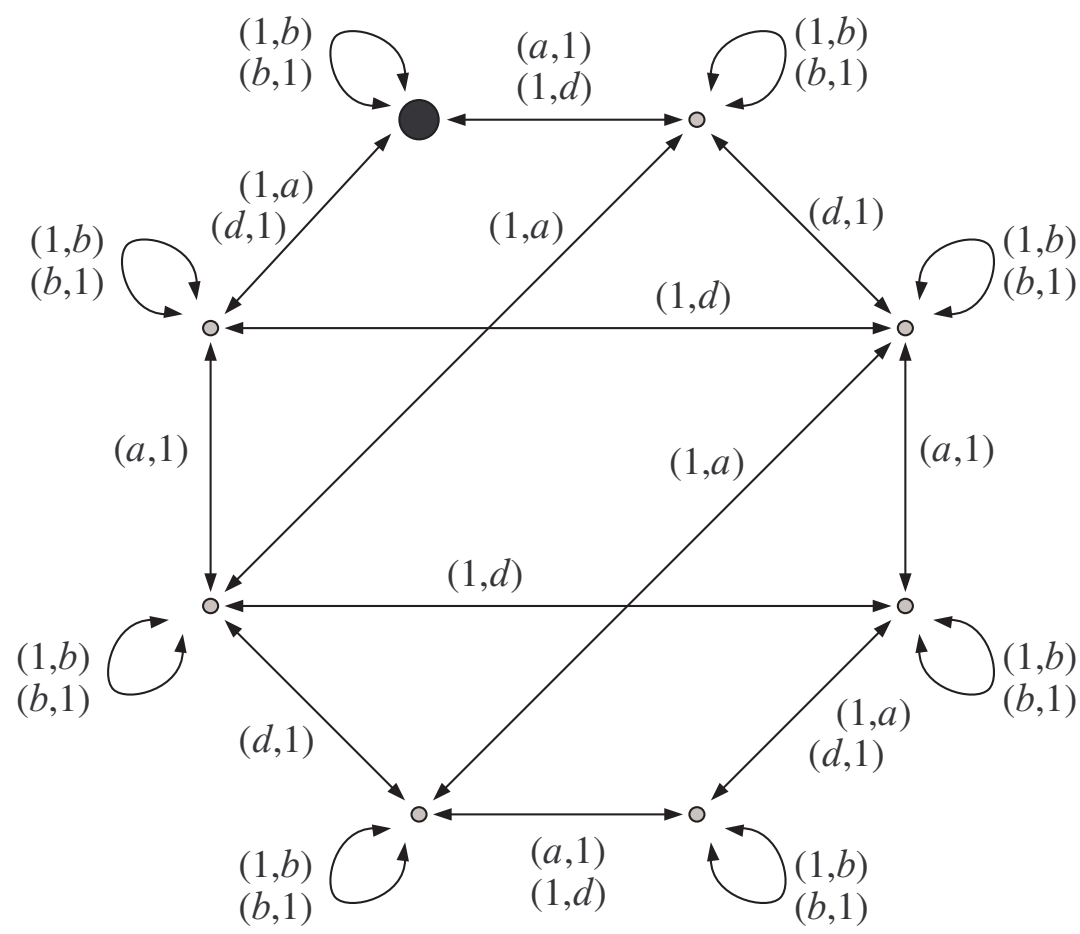

Figure 3. Schreier coset graph of $\psi\left(\operatorname{St}_{\Gamma}(1)\right) \leq \Gamma \times \Gamma$ relative to the generating set $\{(1, a),(1, b),(1, d),(a, 1),(b, 1),(d, 1)\}$. The big black dot corresponds to the coset $\operatorname{St}_{\Gamma}(1)$.

Lemma 2.9. Let $\left(u_{0}, u_{1}\right),\left(v_{0}, v_{1}\right) \in \Gamma \times \Gamma$ be such that $K u_{0}=K v_{0}$ and $K u_{1}=$ $K v_{1}$. If there exists $u \in \Gamma$ such that $\psi(u)=\left(u_{0}, u_{1}\right)$, then there exists $v \in \Gamma$ such that $\psi(v)=\left(v_{0}, v_{1}\right)$. Moreover, $K u=K v$.

Proof. Indeed, let $u_{0}=k_{0} v_{0}, u_{1}=k_{1} v_{1}$ for some $k_{1}, k_{2} \in K$. Then

$$
\left(u_{0}, u_{1}\right)=\left(k_{0} v_{0}, k_{1} v_{1}\right)=\left(k_{0}, 1\right)\left(1, k_{1}\right)\left(v_{0}, v_{1}\right) \text {. }
$$

By Lemma 2.7, $\left(k_{0}, 1\right)$ and $\left(1, k_{1}\right)$ have pre-images in $K$ under $\psi$. Therefore if $\left(u_{0}, u_{1}\right)$ has a pre-image in $\operatorname{St}_{\Gamma}(1)$ then $\left(v_{0}, v_{1}\right)$ also has a pre-image in $\operatorname{St}_{\Gamma}(1)$ and these pre-images lie in the same $K$-coset, as required.

Table 1 below describes completely the $K$-cosets of the pre-images $w$ under $\psi$ of pairs $\left(w_{0}, w_{1}\right)$ of elements from $\Gamma \times \Gamma$ if the cosets of the components $w_{0}, w_{1}$ are given (here numbers $i$ are the indices of the representatives $g_{i}$ of the cosets of $K$ ).

Lemma 2.10. Let $v_{0}, v_{1} \in \Gamma, K v_{0}=K g_{i}$ and $K v_{1}=K g_{j}$. Then there exists $v \in \operatorname{St}_{\Gamma}(1)$ such that $v=\left(v_{0}, v_{1}\right)$ if and only if the pair $(i, j)$ is listed in the Table 1. 


\begin{tabular}{|l|c||l|c||l|c||l|l|}
\hline$\left(w_{0}, w_{1}\right)$ & $w$ & $\left(w_{0}, w_{1}\right)$ & $w$ & $\left(w_{0}, w_{1}\right)$ & $w$ & $\left(w_{0}, w_{1}\right)$ & $w$ \\
\hline$(0,0)$ & 0 & $(8,0)$ & 5 & $(4,4)$ & 0 & $(12,4)$ & 5 \\
\hline$(0,8)$ & 1 & $(8,8)$ & 4 & $(4,12)$ & 1 & $(12,12)$ & 4 \\
\hline$(1,7)$ & 13 & $(9,7)$ & 8 & $(5,3)$ & 13 & $(13,3)$ & 8 \\
\hline$(1,15)$ & 12 & $(9,15)$ & 9 & $(5,11)$ & 12 & $(13,11)$ & 9 \\
\hline$(2,6)$ & 4 & $(10,6)$ & 1 & $(6,2)$ & 4 & $(14,2)$ & 1 \\
\hline$(2,14)$ & 5 & $(10,14)$ & 0 & $(6,10)$ & 5 & $(14,10)$ & 0 \\
\hline$(3,5)$ & 9 & $(11,5)$ & 12 & $(7,1)$ & 9 & $(15,1)$ & 12 \\
\hline$(3,13)$ & 8 & $(11,13)$ & 13 & $(7,9)$ & 8 & $(15,9)$ & 13 \\
\hline
\end{tabular}

Table 1

Proof. By Lemma 2.9 the answer to the question whether a pair $\left(u_{0}, u_{1}\right)$ has a preimage in $\operatorname{St}_{\Gamma}(1)$ under $\psi$ depends only on the coset $(K \times K)\left(u_{0}, u_{1}\right)$, which is completely determined by the cosets $K u_{0}, K u_{1}$ of the components. Therefore, it suffices to check which of the pairs $\left(g_{i}, g_{j}\right), i, j=0, \ldots, 15$, lie in the subgroup $\psi\left(\operatorname{St}_{\Gamma}(1)\right)$. This can be easily done using the Schreier coset graph for $\psi\left(\operatorname{St}_{\Gamma}(1)\right.$; see Figure 3 .

\section{Splittings}

In this section for a word $w \in \mathcal{R}^{e}$ we study the metric properties of the splitting $w \rightarrow\left(w_{0}, w_{1}\right)$, where $w_{i}=\operatorname{red}\left(\phi_{i}(w)\right), i=1,2$. Namely, following [1], we study relations between norms (i.e., weighted lengths) of $w, w_{0}, w_{1}$.

Recall that

$$
\begin{aligned}
b & =(a, c), & & a b a=(c, a), \\
c & =(a, d), & & a c a=(d, a), \\
d & =(1, b), & & \text { ada }=(b, 1) .
\end{aligned}
$$

Let $\gamma_{a}, \gamma_{b}, \gamma_{c}, \gamma_{d}$ be fixed positive real values, termed weights. For a word $w \in X^{*}$ the number

$$
\|w\|=\gamma_{a}|w|_{a}+\gamma_{b}|w|_{b}+\gamma_{c}|w|_{c}+\gamma_{d}|w|_{d}
$$

is called the norm of $w$. The length $|w|$ is a special case of the norm when $\gamma_{a}=$ $\gamma_{b}=\gamma_{c}=\gamma_{d}=1$. In the following lemma we collect some simple properties of the norm $\|\cdot\|$.

Lemma 3.1. Let $u, v, w \in X^{*}$. Then the following holds:

1) $\min \left\{\gamma_{a}, \gamma_{b}, \gamma_{c}, \gamma_{d}\right\} \cdot|w| \leq\|w\| \leq \max \left\{\gamma_{a}, \gamma_{b}, \gamma_{c}, \gamma_{d}\right\} \cdot|w|$.

2) $\|u v\|=\|u\|+\|v\|$. 
3) If the numbers $\gamma_{b}, \gamma_{c}, \gamma_{d}$ satisfy the triangular inequality then

$$
\|\operatorname{red}(w)\| \leq\|w\|
$$

Proof. Straightforward verification.

We define the weights $\gamma_{a}, \gamma_{d}, \gamma_{c}, \gamma_{b}$ which we are using for the remainder of the paper. Let $\alpha$ be the unique real root of the polynomial $2 x^{3}-x^{2}-x-1$,

$$
\alpha \in(1.233751,1.233752) \approx 1.23375 \text {. }
$$

Put

$$
\begin{aligned}
& \gamma_{a}=\alpha^{2}+\alpha-1 \in(1.755892,1.755896) \approx 1.7559, \\
& \gamma_{b}=2, \\
& \gamma_{c}=\alpha^{2}-\alpha+1 \in(1.28839,1.288392) \approx 1.288, \\
& \gamma_{d}=-\alpha^{2}+\alpha+1 \in(0.711608,0.71161) \approx 0.712 .
\end{aligned}
$$

Obviously, the weights $\gamma_{a}, \gamma_{d}, \gamma_{c}, \gamma_{b}$ satisfy the triangle inequality. Note that, up to a multiplicative constant, they are the same as the weights used in [1].

Lemma 3.2. The following equalities hold:

$$
\begin{aligned}
\|a\|+\|b\| & =\alpha(\|a\|+\|c\|), \\
\|a\|+\|c\| & =\alpha(\|a\|+\|d\|), \\
\|a\|+\|d\| & =\alpha\|b\| .
\end{aligned}
$$

Remark 3.3. The choice of the weights $\gamma_{a}, \gamma_{b}, \gamma_{c}, \gamma_{d}$ is optimal in the following sense: the value of $\alpha$ is maximal for the weights satisfying the triangle inequality (for $\left.\gamma_{b}, \gamma_{c}, \gamma_{d}\right)$ and relations in (10).

The following results establish some relations between $\|w\|$ and $\left\|w_{0}\right\|,\left\|w_{1}\right\|$ (cf. [1], Proposition 5).

Lemma 3.4. Let $w \in \mathcal{R}^{e}$ and $w_{i}=\operatorname{red}\left(\psi_{i}(w)\right)$ for $i=0,1$. Then the following holds:

1) If $w$ is of the form $* a * a \ldots * a$ or $a * a * \ldots a *$ (where stars stand for letters $b, c, d)$ then $\alpha\left(\left\|w_{0}\right\|+\left\|w_{1}\right\|\right) \leq\|w\|$.

2) If $w$ is of the form $* a * a \ldots *$ then $\alpha\left(\left\|w_{0}\right\|+\left\|w_{1}\right\|\right) \leq\|w\|+\|a\|$.

3) If $w$ is of the form $a * a \ldots *$ a then $\alpha\left(\left\|w_{0}\right\|+\left\|w_{1}\right\|\right) \leq\|w\|-\|a\|$.

Proof. To prove 1) suppose that $w$ is in the form $* a * a \ldots * a$. If $w=* \cdot a * a$ then the routine case by case verification, based on (9) and (10), shows that

$$
\alpha\left(\left\|w_{0}\right\|+\left\|w_{1}\right\|\right) \leq \alpha\left(\left\|\psi_{0}(w)\right\|+\left\|\psi_{1}(w)\right\|\right)=\|w\| .
$$


In general $w$ can be presented as a product of factors of the type $* \cdot a * a$ :

$$
w=\left(x_{1} \cdot a y_{1} a\right) \ldots\left(x_{k} \cdot a y_{k} a\right)
$$

where $x_{i}, y_{i} \in\{b, c, d\}, i=1, \ldots, k$. In this case Lemma 3.1, item 2), gives (using the fact that $\psi_{0}, \psi_{1}$ are homomorphisms)

$$
\begin{aligned}
\alpha\left(\left\|\psi_{0}(w)\right\|+\left\|\psi_{1}(w)\right\|\right) & =\alpha\left(\sum_{i=1}^{k}\left\|\psi_{0}\left(x_{i} \cdot a y_{i} a\right)\right\|+\sum_{i=1}^{k}\left\|\psi_{1}\left(x_{i} \cdot a y_{i} a\right)\right\|\right) \\
& =\sum_{i=1}^{k}\left\|x_{i} \cdot a y_{i} a\right\|=\mid w \| .
\end{aligned}
$$

Hence, by Lemma 3.1, item 3), $\alpha\left(\left\|w_{0}\right\|+\left\|w_{1}\right\|\right) \leq\|w\|$, as claimed.

To show 2) observe first that for $w=* a * a *$ one has

$$
\alpha\left(\left\|w_{0}\right\|+\left\|w_{1}\right\|\right) \leq\|w\|+\|a\| .
$$

Now the result follows from this and the case 1) above.

To see 3 ) it suffices to notice that for $w=a * a$

$$
\alpha\left(\left\|\psi_{0}(w)\right\|+\left\|\psi_{1}(w)\right\|\right) \leq\|w\|-\|a\|
$$

and then apply an argument as above.

Corollary 3.5. Let $w \in \mathcal{R}$. For $i=0,1$ put

$$
w_{i}= \begin{cases}\operatorname{red}\left(\psi_{i}(w)\right) & \text { if } w \in \mathcal{R}^{e} \\ \operatorname{red}\left(\psi_{i}(w a)\right) & \text { if } w \notin \mathcal{R}^{e}\end{cases}
$$

Then

$$
\alpha\left(\left\|w_{0}\right\|+\left\|w_{1}\right\|\right) \leq\|w\|+\|a\| .
$$

Proof. If $w \in \mathcal{R}^{e}$ then the result follows directly from Lemma 3.4. Suppose that $w \notin \mathcal{R}^{e}$. There are two cases to consider. Case 1. $w$ ends on a letter from $\{b, c, d\}$, i.e., $w=u \cdot *$. Then $w a=u \cdot * \cdot a$ and if $u$ starts with $*$ then by Lemma 3.4, item 1),

$$
\alpha\left(\left\|w_{0}\right\|+\left\|w_{1}\right\|\right) \leq\|w a\|=\|w\|+\|a\|,
$$

as required. Otherwise, $u$ starts with $a$, then by Lemma 3.4, item 3),

$$
\alpha\left(\left\|w_{0}\right\|+\left\|w_{1}\right\|\right) \leq\|w a\|-\|a\|=\|w\|
$$

which implies the result. 
Proposition 3.6. Let $w \in \mathcal{R}$. For $i=0,1$ put

$$
w_{i}= \begin{cases}\operatorname{red}\left(\psi_{i}(w)\right) & \text { if } w \in \mathcal{R}^{e} \\ \operatorname{red}\left(\psi_{i}(w a)\right) & \text { if } w \notin \mathcal{R}^{e}\end{cases}
$$

Then the following holds:

1) If $\|w\| \geq 9$ then $\frac{\|w\|}{\left\|w_{0}\right\|+\left\|w_{1}\right\|} \geq 1.03$.

2) If $\|w\| \geq 200$ then $\frac{\|w\|}{\left\|w_{0}\right\|+\left\|w_{1}\right\|} \geq 1.22$.

Proof. By Corollary 3.5,

$$
\alpha\left(\left\|w_{0}\right\|+\left\|w_{1}\right\|\right) \leq\|w\|+\|a\| .
$$

Hence, if $\|a\|<0.01\|w\|$, which is the case when $\|w\| \geq 200$, then the second inequality holds. Similarly, if $\|w\| \geq 9$ then the first inequality holds.

\section{The conjugacy problem in the Grigorchuk group}

In this section we prove that the conjugacy problem $(\mathrm{CP})$ in $\Gamma$ has a polynomial time decision algorithm.

Lemma 4.1. If $u, v \in X^{*}$ are conjugate in $\Gamma$ then $u \in \mathrm{St}_{\Gamma}(1) \Longleftrightarrow v \in \mathrm{St}_{\Gamma}(1)$.

Proof. Follows from Lemma 2.1 and the definition of conjugate elements.

The next lemma describes behavior of conjugation relative to the splittings $w \rightarrow$ $\left(w_{0}, w_{1}\right)$. Below we frequently use the same notation for a word from $X^{*}$ and the element it represents in $\Gamma$ since it is clear from the context what is meant.

Lemma 4.2 ([9]). Let $u, v, x \in X^{*}$. Then the following holds in $\Gamma$ :

(S1) If $u, v, x \in \mathrm{St}_{\Gamma}(1)$ and $u=\left(u_{0}, u_{1}\right), v=\left(v_{0}, v_{1}\right), x=\left(x_{0}, x_{1}\right)$ then

$$
u=x^{-1} v x \Longleftrightarrow\left\{\begin{array}{l}
u_{0}=x_{0}^{-1} v_{0} x_{0} \\
u_{1}=x_{1}^{-1} v_{1} x_{1}
\end{array}\right.
$$

(S2) If $u, v, x a \in \mathrm{St}_{\Gamma}(1)$ and $u=\left(u_{0}, u_{1}\right), v=\left(v_{0}, v_{1}\right)$, xa $=\left(x_{0}, x_{1}\right)$ then

$$
u=x^{-1} v x \Longleftrightarrow\left\{\begin{array}{l}
u_{0}=x_{1}^{-1} v_{1} x_{1}, \\
u_{1}=x_{0}^{-1} v_{0} x_{0}
\end{array}\right.
$$


(N1) If ua, va, $x \in \mathrm{St}_{\Gamma}(1)$ and $u a=\left(u_{0}, u_{1}\right)$, va $=\left(v_{0}, v_{1}\right), x=\left(x_{0}, x_{1}\right)$ then

$$
u=x^{-1} v x \Longleftrightarrow\left\{\begin{array}{l}
u_{0} u_{1}=x_{0}^{-1} v_{0} v_{1} x_{0}, \\
x_{1}=v_{1} x_{0} u_{1}^{-1}
\end{array}\right.
$$

(N2) If $u a, v a, x a \in \mathrm{St}_{\Gamma}(1)$ and $u a=\left(u_{0}, u_{1}\right), v a=\left(v_{0}, v_{1}\right), x a=\left(x_{0}, x_{1}\right)$ then

$$
u=x^{-1} v x \Longleftrightarrow\left\{\begin{array}{l}
u_{1} u_{0}=x_{0}^{-1} v_{0} v_{1} x_{0}, \\
x_{1}=v_{1} x_{0} u_{0}^{-1}
\end{array}\right.
$$

Proof. (S1) and (S2) immediately follow from (3) and (4).

To see (N1) observe first that $u=u a a, v=v a a$ and so:

$$
\begin{aligned}
u=x^{-1} v x & \Longleftrightarrow\left(u_{0}, u_{1}\right) a=\left(x_{0}^{-1}, x_{1}^{-1}\right)\left(v_{0}, v_{1}\right) a\left(x_{0}, x_{1}\right) \\
& \Longleftrightarrow\left(u_{0}, u_{1}\right)=\left(x_{0}^{-1}, x_{1}^{-1}\right)\left(v_{0}, v_{1}\right) a\left(x_{0}, x_{1}\right) a \\
& \Longleftrightarrow\left(u_{0}, u_{1}\right)=\left(x_{0}^{-1}, x_{1}^{-1}\right)\left(v_{0}, v_{1}\right)\left(x_{1}, x_{0}\right) .
\end{aligned}
$$

This implies the equalities

$$
u_{0}=x_{0}^{-1} v_{0} x_{1}, \quad u_{1}=x_{1}^{-1} v_{1} x_{0}
$$

Multiplying the equalities (11) one gets $u_{0} u_{1}=x_{0}^{-1} v_{0} v_{1} x_{0}-$ the first equality in (N1). Now from this equality one gets $x_{0}^{-1} v_{0}=u_{0} u_{1} x_{0}^{-1} v_{1}^{-1}$. Substituting this into the first equality in (11) gives (after standard manipulations) the second equality $x_{1}=v_{1} x_{0} u_{1}^{-1}$ of $(\mathrm{N} 1)$, as required. It is straightforward to prove the converse. A similar argument proves (N2).

For a pair of elements $u, v \in \Gamma$ define a set

$$
Q(u, v)=\left\{g_{i} \mid \text { there exists } x \in \Gamma \text { such that } u=x^{-1} v x \text { and } K x=K g_{i}\right\},
$$

where $g_{0}, \ldots, g_{15}$ are $K$-coset representatives of $\Gamma$ chosen above. Clearly, $u$ and $v$ are conjugate in $\Gamma$ if and only if $Q(u, v) \neq \varnothing$.

The following is a key lemma in the solution of the conjugacy problem in $\Gamma$ (see [9]).

Lemma 4.3. Let $u, v \in X^{*}$. Then the following holds:

1) If $u=\left(u_{0}, u_{1}\right), v=\left(v_{0}, v_{1}\right) \in \mathrm{St}_{\Gamma}(1)$ then

$$
Q(u, v)=\psi^{-1}\left[Q\left(u_{0}, v_{0}\right) \times Q\left(u_{1}, v_{1}\right)\right] \cup \psi^{-1}\left[Q\left(u_{1}, v_{0}\right) \times Q\left(u_{0}, v_{1}\right)\right] .
$$

Moreover, it takes constant time to compute $Q(u, v)$ if the sets $Q\left(u_{0}, v_{0}\right)$, $Q\left(u_{1}, v_{1}\right), Q\left(u_{0}, v_{1}\right)$ and $Q\left(u_{1}, v_{0}\right)$ are given. 
2) If $u a=\left(u_{0}, u_{1}\right), v a=\left(v_{0}, v_{1}\right) \in \mathrm{St}_{\Gamma}(1)$ then

$$
\begin{aligned}
Q(u, v)= & \psi^{-1}\left\{\left(g_{i}, g_{j}\right) \mid g_{i} \in Q\left(u_{0} u_{1}, v_{0} v_{1}\right) \text { and } K g_{j}=K v_{1} g_{i} u_{1}^{-1}\right\} \\
& \cup \psi^{-1}\left\{\left(g_{i}, g_{j}\right) \mid g_{i} \in Q\left(u_{1} u_{0}, v_{0} v_{1}\right) \text { and } K g_{j}=K v_{1} g_{i} u_{0}^{-1}\right\} .
\end{aligned}
$$

Moreover, it takes constant time to compute $Q(u, v)$ if the sets $Q\left(u_{0} u_{1}, v_{0} v_{1}\right)$ and $Q\left(u_{1} u_{0}, v_{0} v_{1}\right)$ are given.

Proof. 1) follows directly from Lemma 4.2, items (S1) and (S2), and Lemma 2.10.

Similarly, 2) follows from Lemma 4.2, items (N1) and (N2), and Lemma 2.10.

Lemma 4.3 suggests a branching decision algorithm for the CP (abbreviated to BDAC) in $\Gamma$. The main idea of BDAC is the following: to check whether two given words $u, v \in X^{*}$ are conjugate or not in $\Gamma$ it suffices to verify if the set $Q(u, v)$ is empty or not. Hence the conjugacy problem for elements $u, v$ is reduced to computing the set $Q(u, v)$. To compute $Q(u, v)$ we are going to compute first the sets $Q\left(u^{\prime}, v^{\prime}\right)$ for a finite set of pairs $\left(u^{\prime}, v^{\prime}\right)$ that occur in the branching process. To see how the algorithm works let $\left(u^{\prime}, v^{\prime}\right)$ be a current pair that occurs in BDAC. There are four cases to consider:

(BDAC0) If $\left|u^{\prime}\right|,\left|v^{\prime}\right| \leq 1$ then we use precomputed sets $Q\left(u^{\prime}, v^{\prime}\right)$. We compute them later in Lemmas 4.7, 4.8, 4.9, 4.10 and 4.11,

(BDAC1) If one of $u^{\prime}, v^{\prime}$ is in $\mathrm{St}_{\Gamma}(1)$ and the other is not then by Lemma 4.1 the set $Q\left(u^{\prime}, v^{\prime}\right)$ is empty, in which case we mark the current pair $\left(u^{\prime}, v^{\prime}\right)$ by $\emptyset$.

(BDAC2) If $u^{\prime}=\left(u_{0}^{\prime}, u_{1}^{\prime}\right), v=\left(v_{0}^{\prime}, v_{1}^{\prime}\right) \in \mathrm{St}_{\Gamma}(1)$ then, by Lemma 4.3 , case 1$)$, computation of $Q\left(u^{\prime}, v^{\prime}\right)$ reduces to computing the sets $Q\left(u_{0}^{\prime}, v_{0}^{\prime}\right), Q\left(u_{1}^{\prime}, v_{1}^{\prime}\right)$, $Q\left(u_{0}^{\prime}, v_{1}^{\prime}\right), Q\left(u_{1}^{\prime}, v_{0}^{\prime}\right)$.

(BDAC3) If $u^{\prime} a=\left(u_{0}^{\prime}, u_{1}^{\prime}\right), v^{\prime} a=\left(v_{0}^{\prime}, v_{1}^{\prime}\right) \in \mathrm{St}_{\Gamma}(1)$ then, by Lemma 4.3 , case 2), computation of $Q\left(u^{\prime}, v^{\prime}\right)$ reduces to computing the sets $Q\left(u_{0}^{\prime} u_{1}^{\prime}, v_{0}^{\prime} v_{1}^{\prime}\right)$ and $Q\left(u_{1}^{\prime} u_{0}^{\prime}, v_{0}^{\prime} v_{1}^{\prime}\right)$.

Thus, the process either assigns $\emptyset$ to the current pair of words or branches at the pair (with four or two branches, depending on the case at hand). By Proposition 3.6, each branching results in pairs of words with smaller norm, so the process eventually terminates in finitely many steps.

To study the complexity of BDAC it is convenient to break it into two stages.

Stage 1 (Construction of the decision conjugacy tree $T_{u, v}$ ). At the first stage, on an input $u, v \in X^{*} \mathrm{BDAC}$ constructs a finite labeled rooted tree $T_{u, v}$, where every vertex is a pair of words $\left(u_{i}, v_{i}\right)$ from $\mathcal{R}$. Each vertex has degree at most four and some of them are decorated with the symbol $\emptyset$. The pair $(u, v)$ is at the root of $T_{u, v}$. The construction of $T_{u, v}$ follows the rules BDAC0-BDAC3. Namely, if $\left(u^{\prime}, v^{\prime}\right)$ is a current node such that $\left(u^{\prime}, v^{\prime}\right)$ falls into the case BDAC0 or BDAC1 then we leave this node as a leaf in $T_{u, v}$. If $\left(u^{\prime}, v^{\prime}\right)$ falls into the case BDAC2 then the algorithm 
constructs four children nodes $\left(u_{0}^{\prime}, v_{0}^{\prime}\right),\left(u_{1}^{\prime}, v_{1}^{\prime}\right),\left(u_{0}^{\prime}, v_{1}^{\prime}\right)$ and $\left(u_{1}^{\prime}, v_{0}^{\prime}\right)$ according to BDAC2 (see Figure $4 \mathrm{a})$ ). If $\left(u^{\prime}, v^{\prime}\right)$ falls into the case BDAC 3 then the algorithm constructs two children nodes $\left(u_{0}^{\prime} u_{1}^{\prime}, v_{0}^{\prime} v_{1}^{\prime}\right)$ and $\left(u_{1}^{\prime} u_{0}^{\prime}, v_{0}^{\prime} v_{1}^{\prime}\right)$ as described in BDAC3 (see Figure $4 \mathrm{~b}$ )). Notice that it takes linear time in $|u|+|v|$ to assign $\varnothing$ to a given node or to produce its children.
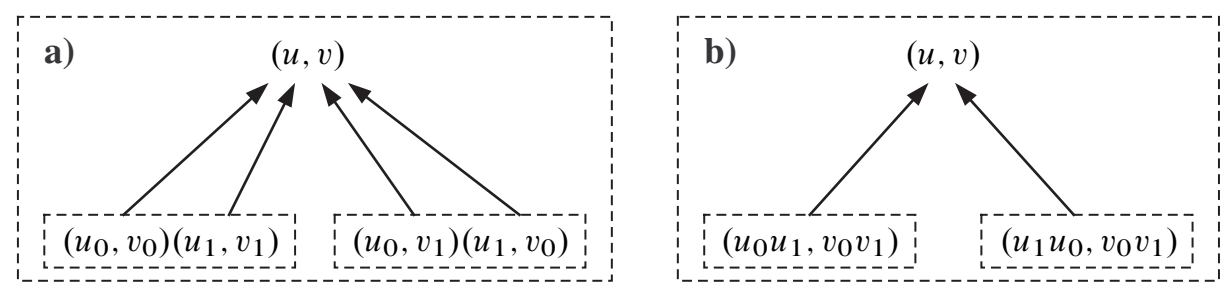

Figure 4. Two types of nodes in $T_{u, v}$ corresponding to cases BDAC2 and BDAC3.

Stage 2 (Computing the sets $Q\left(u_{i}, v_{i}\right)$ involved in $\left.T_{u, v}\right)$. At the second stage, BDAC, going from the leafs of the tree $T_{u, v}$ to the root $(u, v)$, assigns to each vertex $\left(u^{\prime}, v^{\prime}\right)$ of $T_{u, v}$ the set $Q\left(u^{\prime}, v^{\prime}\right)$, computed as described in Lemma 4.3. It is left to assign the set $Q\left(u^{\prime}, v^{\prime}\right)$ to each leaf $\left(u^{\prime}, v^{\prime}\right)$ in $T_{u, v}$. If the leaf $\left(u^{\prime}, v^{\prime}\right)$ corresponds to the case BDAC0 then we use the precomputed sets $Q\left(u^{\prime}, v^{\prime}\right)$. If the leaf $\left(u^{\prime}, v^{\prime}\right)$ corresponds to the case BDAC1 then $Q\left(u^{\prime}, v^{\prime}\right)=\emptyset$. It takes constant time to assign the set $Q\left(u^{\prime}, v^{\prime}\right)$ to a vertex $\left(u^{\prime}, v^{\prime}\right)$, indeed, it is obvious for the leaves and follows from Lemma 4.3 for the other vertices.

It is clear from the description of BDAC that the time required for BDAC to stop and to obtain the answer on an input $(u, v)$ can be estimated from above by the time to construct the tree $T_{u, v}$ and the time spent on labeling the vertices. Using the standard breadth-first algorithm the construction of the tree $T_{u, v}$ takes linear time in the size $\left|T_{u, v}\right|$ multiplied by the time spent at every vertex, so altogether is bounded from the above by $\left|T_{u, v}\right|(|u|+|v|)$. To get the polynomial estimate on the time complexity of BDAC we show below that the size of the tree $T_{u, v}$ is polynomial in terms of $|u|+|v|$.

The next result shows that for any words $u, v \in \mathcal{R}$ the size $\left|T_{u, v}\right|$ of the tree $T_{u, v}$ is polynomial in terms of $|u|$ and $|v|$ and gives estimates on the size.

Lemma 4.4. Let $u, v \in \mathcal{R}$. If $\|v\|<9$ and $\|u\|<9$ then the size of the tree $T_{u, v}$ is not greater than 42 .

Proof. The set of pairs of words $(u, v)$ such that $\|v\|<9$ and $\|u\|<9$ is finite, though relatively large, and so the statement of the lemma can be checked by a straightforward verification.

Nevertheless, it is possible to check the correctness of the statement by hand. Notice that for any child $\left(u^{\prime}, v^{\prime}\right)$ of $(u, v)$ the element $u^{\prime}$ depends only on the element $u$ and the element $v^{\prime}$ depends only on the element $v$. In other words, one can assume that the left and right words in the vertices are independent of each other, so that one 
can consider each of them separately. Table 2 contains all 95 words $w$ of norm up to 9. For each $w$ it specifies the children of $w$ (defined as in cases BDAC2 and BDAC3) and the size of the tree necessary to reach words of lengths up to 1 at the leaves. The greatest size is 21 and hence, since we have 2 coordinates, the size of $T_{u, v}$ does not exceed $2 \cdot 21=42$.

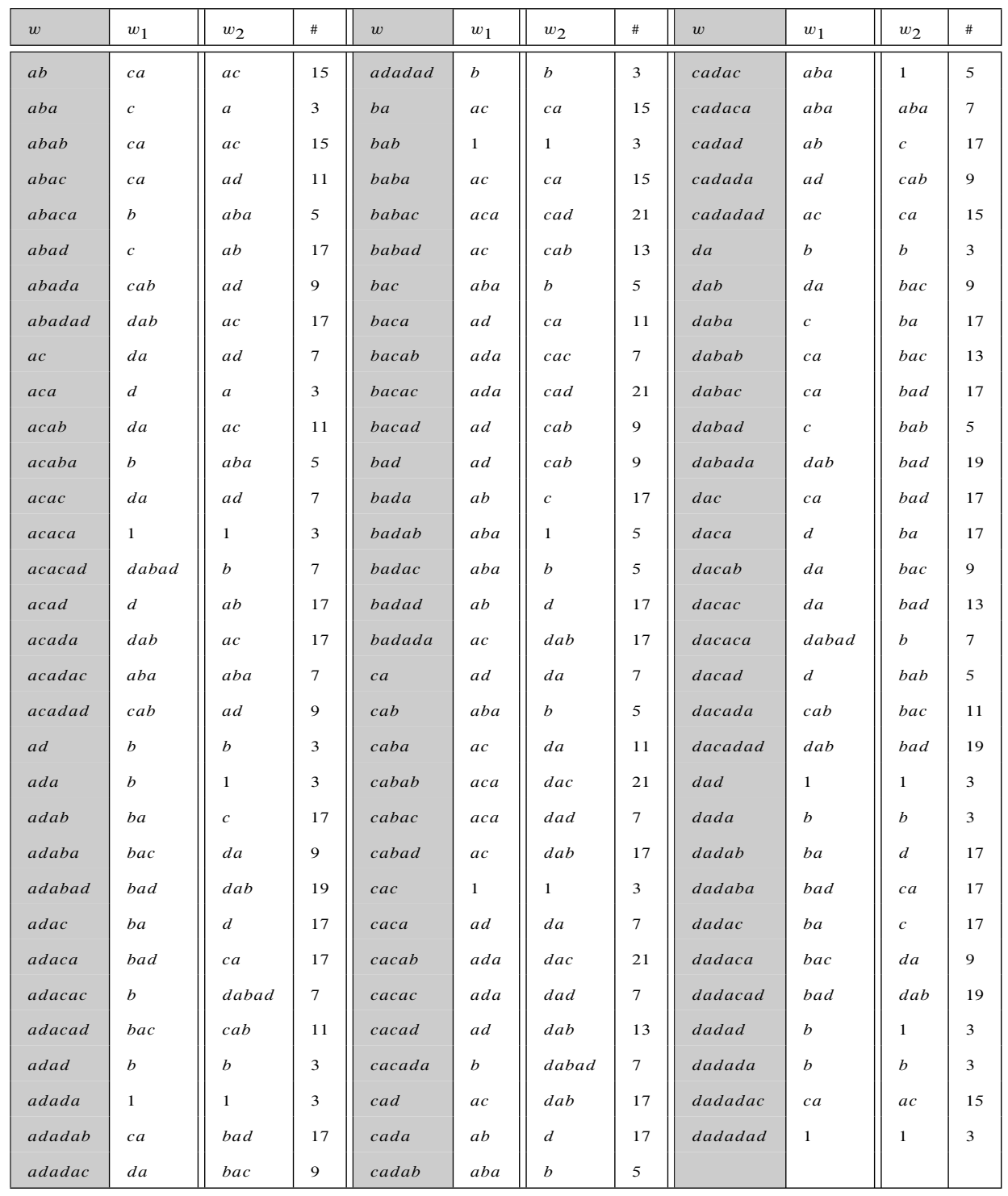

Table 2 
Proposition 4.5. Let $u, v \in \mathcal{R}$. Then the size of the tree $T_{u, v}$ is bounded by $2^{366}(\max \{2|u|, 2|v|\})^{7}$.

Proof. Let $u, v \in \mathcal{R}$. As in the proof of Lemma 4.4 one can consider the left and right words in the vertices of the tree separately.

By Lemma 3.1, item 1), $\|w\| \leq 2|w|$. Consider an arbitrary branch

$$
\left(u_{0}, v_{0}\right), \ldots,\left(u_{k}, v_{k}\right)
$$

in the tree $T_{u, v}$, starting at the root $\left(u_{0}, v_{0}\right)=(u, v)$ and ending at a leaf $\left(u_{k}, v_{k}\right)$. It follows from Proposition 3.6 that for some $s \leq \log _{1.22} \max \{2|u|, 2|v|\}$ the inequalities $\left\|v_{s}\right\| \leq 200$ and $\left\|u_{s}\right\| \leq 200$ hold, i.e., any branch reaches a pair of words of norm up to 200 in at $\operatorname{most} \log _{1.22} \max \{2|u|, 2|v|\}$ steps. Furthermore, by Proposition 3.6 there exists a number $t \leq \log _{1.03} 200<180$ such that $\left\|v_{s+t}\right\|<9$ and $\left\|u_{s+t}\right\|<9$. By Lemma 4.4 the size of the tree $T_{v_{s+t}, u_{s+t}}$ is not greater than 42 . Thus, since the degree in each node in $T_{u, v}$ is not greater than 4 , it follows that the size of $T_{u, v}$ is bounded by

$$
\begin{aligned}
\left|T_{u, v}\right| & \leq 42 \cdot 4^{180+\log _{1.22} \max \{2|u|, 2|v|\}} \\
& \leq 2^{366}(\max \{2|u|, 2|v|\})^{\log _{1.22} 4} \\
& \leq 2^{366}(\max \{2|u|, 2|v|\})^{7},
\end{aligned}
$$

as claimed.

Theorem 4.6. The conjugacy problem in the Grigorchuk group $\Gamma$ is decidable in $O\left(n^{8}\right)$ time.

Proof. Given two words $u, v \in \mathcal{R}$ the algorithm BDC constructs the tree $T_{u, v}$, the size of which is bounded by $2^{366}(\max \{2|u|, 2|v|\})^{7}$. Processing each of the nodes of the tree requires $O(\max \{|u|,|v|\})$ elementary steps. Thus, the total complexity of the algorithm is bounded by $O\left((\max \{|u|,|v|\})^{8}\right)$.

For completeness we list below the sets $Q(u, v)$ with $|u|,|v| \leq 1$.

Lemma 4.7. The following holds:

$$
\begin{gathered}
Q(a, b)=Q(a, c)=Q(a, b)=\emptyset, \\
Q(1, a)=Q(1, b)=Q(1, c)=Q(1, d)=\emptyset, \\
Q(1,1)=\{0, \ldots, 15\} .
\end{gathered}
$$

Proof. This follows immediately from Lemma 4.1.

Lemma 4.8. $Q(b, c)=Q(b, d)=Q(c, d)=\emptyset$. 
Proof. Since $b, c, d \in \mathrm{St}_{\Gamma}(1)$ by Lemma 4.3 we have

$$
\begin{aligned}
Q(c, d) & =\psi^{-1}[Q(a, 1) \times Q(d, b)] \cup \psi^{-1}[Q(a, b) \times Q(d, 1)] \\
& =\psi^{-1}[\emptyset \times Q(d, b)] \cup \psi^{-1}[Q(a, b) \times \emptyset]=\emptyset .
\end{aligned}
$$

Similarly,

$$
\begin{aligned}
Q(b, d) & =\psi^{-1}[Q(a, 1) \times Q(c, b)] \cup \psi^{-1}[Q(a, b) \times Q(c, 1)] \\
& =\psi^{-1}[\emptyset \times Q(d, b)] \cup \psi^{-1}[Q(a, b) \times \emptyset]=\emptyset .
\end{aligned}
$$

Finally, using the obtained equalities we obtain

$$
\begin{aligned}
Q(b, c) & =\psi^{-1}[Q(a, a) \times Q(c, d)] \cup \psi^{-1}[Q(a, d) \times Q(c, a)] \\
& =\psi^{-1}[Q(a, a) \times \emptyset] \cup \psi^{-1}[\emptyset \times \emptyset]=\emptyset .
\end{aligned}
$$

Lemma 4.9. $Q(a, a)=\{0,3,4,7\}$.

Proof. There are two cases to consider. If $x^{-1} a x=a$ and $x \in \operatorname{St}_{\Gamma}(1)$ then $\psi(x)$ is of the form $(y, y) \in \Gamma \times \Gamma$. It follows from Lemma 2.10 and Table 1 that in this case $K x=K g_{0}$ or $K x=K g_{4}$.

If $x^{-1} a x=a$ and $x \notin \mathrm{St}_{\Gamma}(1)$ then $\psi(x a)$ is of the form $(y, y) a \in \Gamma \times \Gamma$. Using Figure 2 it is easy to find that $K g_{0} a=K g_{7}$ and $K_{g_{4}} a=K_{3}$. Hence $Q(a, a)=\{0,3,4,7\}$.

Lemma 4.10. $Q(b, b)=\{0,1,8,9\}, Q(c, c)=\{0,1,8,9\}$.

Proof. Notice that $1, b, c, d \in C_{\Gamma}(b)$, where $C_{\Gamma}(b)$ denotes the centralizer of $b$ in $\Gamma$. Hence $Q(b, b) \supseteq\{0,1,8,9\}$. On the other hand assume that $x^{-1} b x=b$. Then it is easy to see that $x=\left(x_{0}, x_{1}\right) \in \operatorname{St}_{\Gamma}(1)$ and splitting $b$ into a pair $(a, c)$ we get

$$
Q(b, b)=\psi^{-1}[Q(a, a) \times Q(c, c)] .
$$

We proved in Lemma 4.9 that $Q(a, a)=\{0,3,4,7\}$. Notice that in Table 1 all pairs $(i, j)$ with $i \in\{0,3,4,7\}$ define cosets with numbers $\{0,1,8,9\}$. Hence $Q(b, b) \subseteq$ $\{0,1,8,9\}$.

The proof for $Q(c, c)$ is the same.

Lemma 4.11. $Q(d, d)=\{0,1,4,5,8,9,12,13\}$.

Proof. Notice that $1, b, c, d \in C_{\Gamma}(d)$, where $C_{\Gamma}(d)$ denotes the centralizer of $d$ in $\Gamma$. Hence $Q(d, d) \supseteq\{0,1,8,9\}$ Furthermore, ada, ada $\cdot b, a d a \cdot c, a d a \cdot d \in C_{\Gamma}(d)$. Hence $Q(d, d) \supseteq\{4,5,12,13\}$.

On the other hand assume that $x^{-1} d x=d$. Then it is easy to see that $x=$ $\left(x_{0}, x_{1}\right) \in \mathrm{St}_{\Gamma}(1)$ and splitting $d$ into a pair $(1, b)$ we obtain that

$$
Q(d, d)=\psi^{-1}[Q(1,1) \times Q(b, b)] .
$$


Notice that in Table 1 all pairs $(i, j)$ with $i \in\{0, \ldots, 15\}$ and $j \in\{0,1,8,9\}$ define cosets with numbers $\{0,1,4,5,8,9,12,13\}$. Therefore it follows that $Q(d, d) \subseteq$ $\{0,1,4,5,8,9,12,13\}$.

\section{References}

[1] L. Bartholdi, The growth of Grigorchuk's torsion group. Internat. Math. Res. Notices 1998 (1998), No. 20, 1049-1054. Zbl 0942.20027 MR 1656258

[2] L. Bartholdi, R. I. Grigorchuk, and Z. Šunik, Branch groups. In Handbook of algebra, Vol. 3, North-Holland, Amsterdam 2003, 989-1112. Zbl 1140.20306 MR 2035113

[3] CRyptography And Groups (CRAG) C++ and Python Library. Available at http://www.acc.stevens.edu/downloads.php

[4] M. Garzon and Y. Zalcstein, The complexity of Grigorchuk groups with application to cryptography. Theoret. Comput. Sci. 88 (1991), 83-98. Zbl 0749.68040 MR 1130373

[5] R. I. Grigorchuk, On Burnside's problem on periodic groups. Funktsional. Anal. $i$. Prilozhen. 14 (1980), 53-54; English transl. Funct. Anal. Appl. 14 (1980), 41-43. Zbl 0595.20029 MR 0565099

[6] R. I. Grigorchuk, On growth in group theory. In Proc. Internat. Congr. of Mathematicians (Kyoto, 1990), Vol. 1, Springer-Verlag, Tokyo 1991, 325-338. Zbl 0749.20016 MR 1159221

[7] R. I. Grigorchuk, On a problem of Day on nonelementary amenable groups in the class of finitely presented groups. Mat. Zametki 60 (1996), 774-775; English transl. Math. Notes 60 (1996), 580-582. Zbl 0930.43001 MR 1619929

[8] R. I. Grigorchuk, On the system of defining relations and the Schur multiplier of periodic groups generated by finite automata. In Groups St. Andrews 1997 in Bath, I, London Math. Soc. Lecture Note Ser. 260, Cambridge Univ. Press, Cambridge 1999, 290-317. Zbl 1114.20303 MR 1676626

[9] R. Grigorchuk, Solved and unsolved problems around one group. In Infinite groups: geometric, combinatorial and dynamical aspects, Progr. Math. 248, Birkhäuser, Basel 2005, 117-218. Zbl 1165.20021 MR 2195454

[10] P. de la Harpe, Topics in geometric group theory. Chicago Lectures in Math., The University of Chicago Press, Chicago 2000. Zbl 0965.20025 MR 1786869

[11] G. Higman, Subgroups of finitely presented groups. Proc. Roy. Soc. Ser. A 262 (1961), 455-475. Zbl 0104.02101 MR 0130286

[12] Y. G. Leonov, Conjugacy problem in a class of 2-groups. Mat. Zametki 64 (1998), 573-583; English trasnl. Math. Notes 64 (1998), 496-505. Zbl 0942.20011 MR 1687212

[13] A. I. Mal'tsev, Constructive algebras I. Uspekhi Mat. Nauk 16(1961), No. 3, 3-60; English transl. Russian Math. Surveys 16 (1961), No.3, 77-129. Zbl 0129.25903 MR 0151377

[14] A. G. Myasnikov and A. Ushakov, Random subgroups and analysis of the length-based and quotient attacks. J. Math. Cryptol. 2 (2008), 29—61. Zbl 05309569 MR 2451658 
[15] G. Petrides, Cryptanalysis of the public key cryptosystem based on the word problem on the Grigorchuk groups. In Cryptography and coding, Lecture Notes in Comput. Sci. 2898, Springer, Berlin 2003, 234-244. Zbl 1123.94357 MR 2090936

[16] M. O. Rabin, Computable algebra, general theory and theory of computable fields. Trans. Amer. Math. Soc. 95 (1960), 341-360. Zbl 0156.01201 MR 0113807

[17] A. V. Rozhkov, Conjugacy problem in an automorphism group of an infinite tree. Mat. Zametki 64 (1998), 592-597; English transl. Math. Notes 64 (1998), 513-517. Zbl 0949.20025 MR 1687204

[18] J. S. Wilson and P. A. Zalesskii, Conjugacy separability of certain torsion groups. Arch. Math. (Basel) 68 (1997), 441-449. Zbl 0877.20020 MR 1444655

Received August 27, 2008; revised May 2, 2009

I. Lysenok, Steklov Mathematical Institute, Gubkina str. 8, 119991, Moscow, Russia E-mail: igor.lysenok@gmail.com

A. Myasnikov, Department of Mathematics and Statistics, McGill University, 805 Sherbrooke Street West, Montreal, Quebec, H3A 2K6, Canada

E-mail: amiasnikov@gmail.com

A. Ushakov, Department of Mathematical Sciences, Stevens Institute of Technology, Castle Point on Hudson, Hoboken, NJ 07030, U.S.A.

E-mail: sasha.ushakov@gmail.com 